\title{
Brain Water Diffusion in Normal and Creatine-Supplemented Rats During Transient Global Ischemia
}

\author{
Markus Wick, Hiroyuki Fujimori, Thomas Michaelis, ${ }^{*}$ and Jens Frahm
}

\begin{abstract}
Brain water diffusion in response to transient global ischemia (12 $\mathrm{min}$ ), reperfusion (60 $\mathrm{min})$, and cardiac arrest was monitored by localized proton magnetic resonance spectroscopy. The trace of the apparent diffusion coefficient tensor $\left(A D C_{A v}\right)$ was determined at high temporal resolution $(10 \mathrm{sec})$ to assess the putative neuroprotective potential of oral creatine $(\mathrm{Cr})$ in rats that received $2.2 \mathrm{~g} \mathrm{Cr}$-monohydrate per $\mathrm{kg}$ body weight per day for 10 days $(n=8)$ relative to controls $(n=9)$. Cr-fed rats revealed a statistically significant increase of the cerebral concentration ratio of $\mathrm{Cr}$ to choline-containing compounds $(20 \%)$. The decrease of the $A D C_{A v}$ value during acute ischemia showed a three-phasic behavior in line with energy depletion, cytotoxic edema, and brain cooling. In Cr-fed rats, slightly less severe and mildly delayed diffusion changes during ischemia and similar beneficial trends during early reperfusion did not reach statistical significance. Magn Reson Med 42:798-802, 1999. () 1999 Wiley-Liss, Inc.
\end{abstract}

Key words: magnetic resonance spectroscopy; water diffusion; cerebral metabolism; creatine; cerebral ischemia; neuroprotection

A nimal studies have linked elevated brain creatine $(\mathrm{Cr})$ and phosphocreatine $(\mathrm{PCr})$ to neuroprotection. For example, in hippocampal slices, the availability of $\mathrm{Cr}$ was shown to yield enhanced PCr levels and reduced neuronal death under hypoxic stress most li kely caused by a del ayed decrease of adenosine triphosphate (ATP) (1-3). Under in vivo conditions, localized magnetic resonance spectroscopy (MRS) reveal ed increases of total brain creatine ( $\mathrm{tCr}$ ) levels after oral supplementation of Cr-monohydrate in both rats (4-6) and humans (7). In addition, at least a partial protection of the brain against an impairment of energy metabolism was demonstrated in an animal model of Huntington's disease (5) as well as in hypoxic immature rats (6).

In a preceding proton MRS study of $\mathrm{Cr}$ supplementation in a rat model of transient global ischemia, thetime courses of cerebral metabol ite concentrations such as lactate (Lac) and glucose $(\mathrm{Glc})$ revealed no differences between $\mathrm{Cr}$-fed rats and controls on a ti mescal e of minutes (4). Complementary, diffusion-weighted MRS $(8,9)$ and MRI $(10,11)$ of brain water allows monitoring of ischemia-induced al ter-

\footnotetext{
Biomedizinische NMR Forschungs GmbH am, Max-Planck-Institut für biophysikalische Chemie, Göttingen, Germany.

Hiroyuki Fujimori's present address is Department of Neurosurgery, Institute of Clinical Medicine, University of Tsukuba, 1-1-1 Tennodai, Ibaraki 305, Japan.

${ }^{*}$ Correspondence to: Thomas Michaelis, Ph.D., Biomedizinische NMR Forschungs $\mathrm{GmbH}$ am, Max-Planck-Institut für biophysikalische Chemie, Am Fassberg 11, D-37070 Göttingen, Germany. E-mail: tmichae@gwdg.de

Received 6 May 1999; revised 28 June 1999; accepted 7 July 1999.

(1) 1999 Wiley-Liss, Inc.
}

ations of the apparent diffusion coefficient (ADC) at a temporal resol ution of up to $10 \mathrm{sec}$. The diffusion sensitivity is generally ascribed to the occurrence of cytotoxic cell swelling (cytotoxic edema) which results from the depolarisation of cell membranes and an osmotically driven influx of water. The underlying loss of normal cell ion homeostasis is caused by a failure of the plasma membrane ion pumps which in turn reflects the loss of ATP within regions of severely reduced blood flow (12).

The purpose of this work was to determine whether the rapid diffusion changes following transient global ischemia and cardiac arrest are modulated or even al leviated by oral administration of $\mathrm{Cr}$ using high-speed determinations of the trace of the ADC tensor, i.e., $A D C_{A v}$. The study was based on the hypothesis that el evated $\mathrm{Cr} / \mathrm{PCr}$ levels may delay and/or attenuate the decrease of brain ATP level s and therefore stabilize the energy metabol ism during the early stages of ischemia. A preliminary account has been given in abstract form (13).

\section{MATERIALS AND METHODS}

Studies of male Wistar rats were performed in accordance with German animal-protection laws and approved by the responsible governmental authority. The $\mathrm{Cr}$-fed group ( $n=8,315 \pm 26 \mathrm{~g}$ ) received Cr-monohydrate (Sigma Chemical Co., St. Louis, MO) in thei $r$ feed at a dose of $2.23 \pm 0.12$ $\mathrm{g}$ per $\mathrm{kg}$ body weight per day $(0.5 \%$ in water, $2.0 \%$ in rodent chow) for 10 days, whereas control animals $(n=9$, $319 \pm 50 \mathrm{~g}$ ) were on a normal diet.

Cr-fed rats and controls underwent a protocol consisting of water-suppressed localized proton MRS of cerebral metabolites and subsequent dynamic determinations of $A D C_{A v}$ values obtained from the unsuppressed water signal. Diffusion measurements (100 $\mathrm{min}$ ) were performed before (10 $\mathrm{min})$ and during transient global ischemia (12 min, four-vessel occlusion model), during reperfusion (60 min), and after cardiac arrest (18 min, $\mathrm{KCl}$ injection). Details of the experimental setup, surgical procedure, anesthesia and ventilation, reversible occlusion, and physiologic monitoring were as previously described (4). In particular, there were no statistically significant differences in arterial $\mathrm{P}_{\mathrm{O}_{2}}, \mathrm{P}_{\mathrm{CO}_{2}}, \mathrm{pH}$, mean blood pressure, and plasma Glc level between Cr-fed rats and controls (unpaired t test) or before and after ischemia (paired $t$ test). 
MR measurements were carried out at 2.35 T using a MRBR 4.7/400 mm magnet (Magnex Scientific, A bingdon, England) and a $100 \mathrm{mT} \cdot \mathrm{m}^{-1} \mathrm{~B}-\mathrm{GA} 20$ gradient system driven by ABX-11 electronics (Bruker, Karlsruhe, Germany). Radiofrequency excitation and signal reception were accomplished by a 14-cm Helmholtz coil and a 2-cm surface coil , respecti vel y. Local ized short echo-time proton MR spectra (STEAM, TR/TE/TM = 6000/20/10 msec) were acquired from a $0.245 \mathrm{ml}\left(7 \times 5 \times 7 \mathrm{~mm}^{3}\right)$ volume of interest (VOI) centrally placed in the cortex. Metabolite quantification involved fully automated and user-independent spectral evaluation by LCM odel and cal ibration with respect to the brain water concentration (4).

Diffusion-weighted proton MRS (STEAM, TR/TE/ $\mathrm{TM}=1000 / 140 / 10 \mathrm{msec}$ ) of the water signal from the same VOI was performed at high temporal resolution (10 sec) using three pai rs of nonoverlapping bi polar lobes of orthogonal gradients with the same polarity. Diffusion gradients were placed symmetrically around the TM period to ensure single-shot isotropic diffusion weighting as proposed by Mori and van Zijl (14). The MRS implementation avoids any cross talk between localization and diffusion gradients, i.e., transverse magnetizations are fully refocused before and after the diffusion weighting periods and the effects of background gradients are automati cally compensated. To mi nimize contributions from restricted diffusion, both the gradient duration $\delta$ and the diffusion time $\Delta$ between corresponding gradient lobes were adjusted to $\delta=$ $\Delta=10 \mathrm{msec}$. Respirator-triggered acquisitions of brain water diffusion were based on 10 spectra with variable gradient strengths of up to $90 \mathrm{mT} \cdot \mathrm{m}^{-1}$. Pertinent measurements were obtained continuously during the entire protocol and exploited for online determinations of the trace of the diffusion tensor

$$
A D C_{A v}=\frac{1}{3}\left(A D C_{x x}+A D C_{y y}+A D C_{z z}\right)
$$

from the strength of the water signal in the time domain (10- $\mathrm{Hz}$ line broadening) in accordance to the StejskalTanner equation

$$
\begin{gathered}
A=A_{0} \exp \left(-b_{A v} \cdot A D C_{A v}\right) \\
b_{A v}=n \gamma^{2} G^{2} \delta^{2}\left(\Delta-\frac{1}{3} \delta\right)
\end{gathered}
$$

with $A$ the measured signal strength at diffusion gradient strength $G, A_{0}$ the signal at zero gradient strength, $n$ the number of bipolar gradient pairs $(n=6)$, and $\gamma$ the magnetogyric ratio. Apart from the diffusion weighting of the localization gradients (173 $\mathrm{sec} \cdot \mathrm{mm}^{-2}$ ), the isotropic $b_{\mathrm{Av}}$ val ues were $97,218,387,605,872,1186,1550,1749,1961$, and $2185 \mathrm{sec} \cdot \mathrm{mm}^{-2}$.

Online calculations of $A D C_{A v}$ values were done using a modified Levenberg-Marquard al gorithm (IMSL-C Numerical Libraries Version 1.02, Visual Numerics, Inc., Houston, Texas). The $A_{D C}$ time course was continuously displayed (Gnu-Plot, version 3.5, C. Kelley, T. Williams) to confirm a successful occlusion of the carotid arteries by a corresponding $\mathrm{ADC}_{\mathrm{Av}}$ decrease.

\section{RESULTS}

Figure 1 compares mean proton MR spectra of rat brain in vivo from $\mathrm{Cr}$-fed rats and controls. The visible trends toward elevated $\mathrm{tCr}(7 \%)$ and myo-inositol (Ins, 8\%) are confirmed when using concentration ratios with reference to chol ine-contai ning compounds (Cho). In linewith previous findings (4), Cr-fed rats revealed statistically significant increases (unpaired t tests) of tCr/Cho (20\%) and Ins/Cho (24\%).

Mean time courses of the $\mathrm{ADC}_{\mathrm{Av}}$ in response to transient global ischemia (hashed bar), reperfusion, and cardiac arrest (black bar) are shown in Figure 2 for both $\mathrm{Cr}$-fed rats and controls. The observed $A \mathrm{AC}_{\mathrm{Av}}$ reductions during ischemia (and after cardiac arrest) and its recovery during reperfusion were very similar in both groups. The slightly smaller decrease of the ischemic $A D C_{A v}$ value in the $\mathrm{Cr}$ group was statistically not significant. These findings are supported by the quantitative data summarized in Table 1. Whereas the mean $\mathrm{ADC}_{\mathrm{Av}}$ values for $\mathrm{Cr}$-fed rats and controls are almost identical before and 50-60 min after ischemia, the percentage reductions $\triangle \mathrm{ADC}_{\mathrm{Av}}$ after $12 \mathrm{~min}$ of ischemia and cardiac arrest are slightly less pronounced in $\mathrm{Cr}$-fed rats.
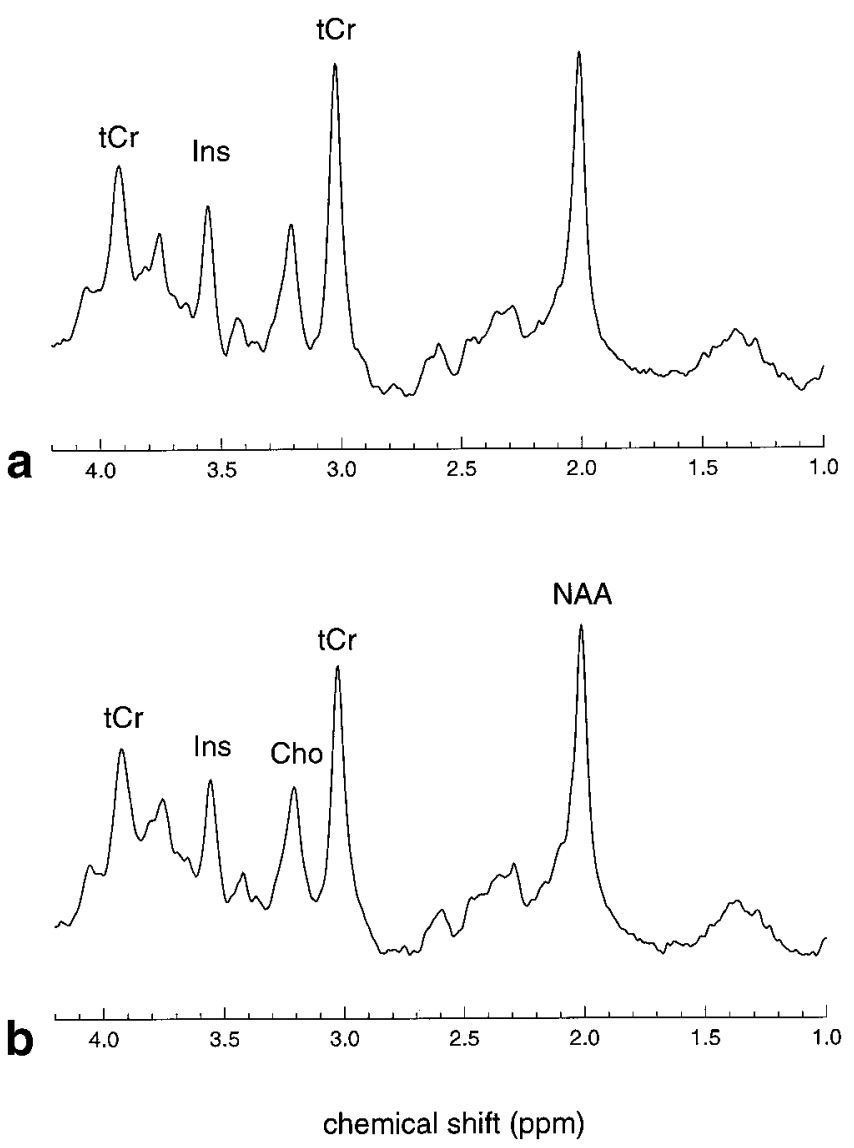

FIG. 1. Mean brain spectra of (a) Cr-fed rats $(n=8)$ and (b) controls $(\mathrm{n}=9)$ obtained by localized proton MRS (STEAM, TR/TE/ $\mathrm{TM}=6000 / 20 / 10 \mathrm{msec}, 0.245 \mathrm{~mL}$ VOI, 32-64 accumulations per animal). Metabolites include $\mathrm{N}$-acetylaspartate (NAA), total creatine (tCr), choline-containing compounds (Cho), and myo-inositol (Ins). Before averaging across animals the individual spectra were scaled in absolute units in proportion to the brain water concentration. 

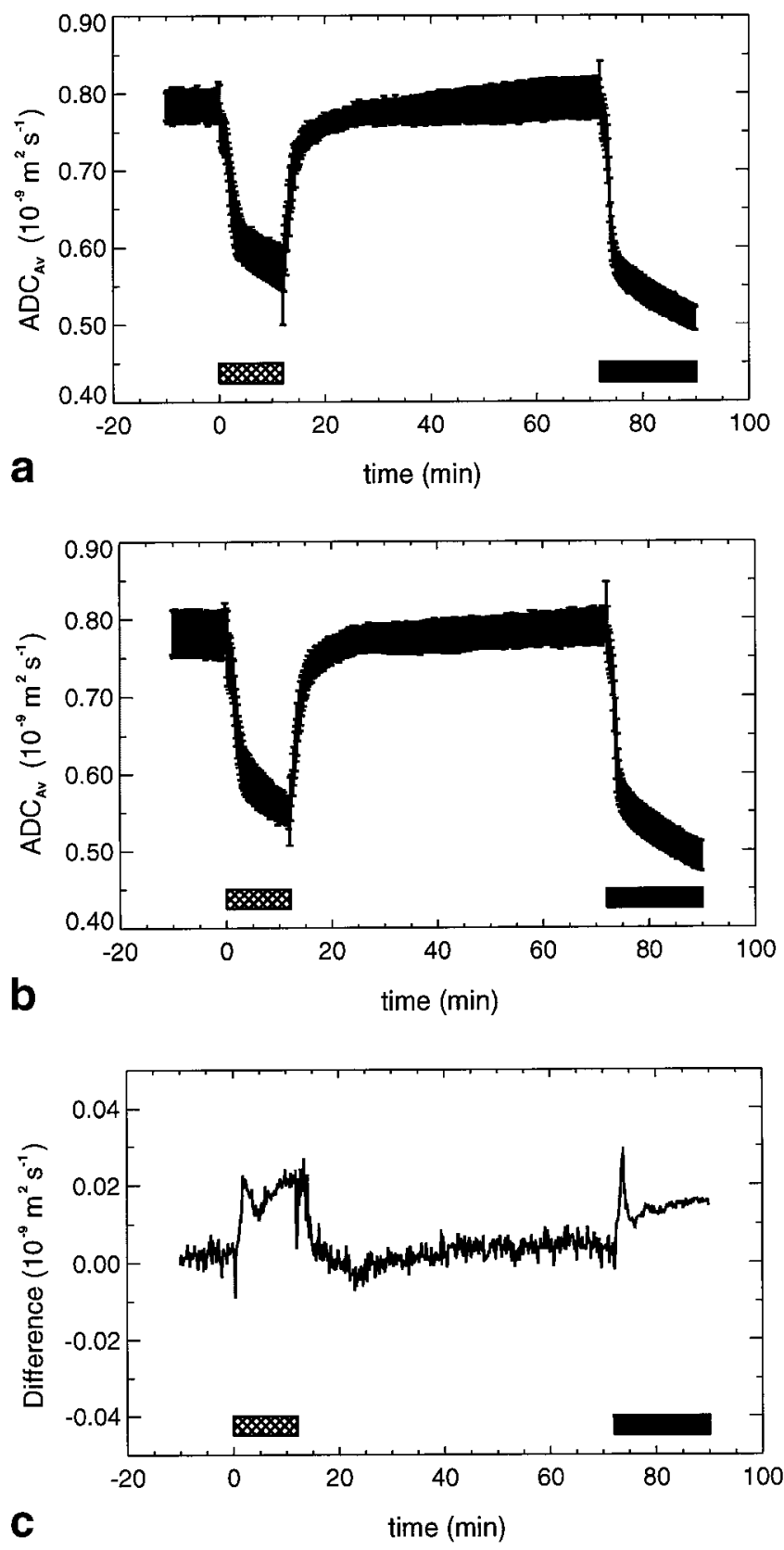

FIG. 2. Mean time courses of the trace of the brain water apparent diffusion coefficient tensor $A D C_{A v}$ (mean $\pm S D$ ) in response to transient global ischemia (hatched bar), reperfusion, and cardiac arrest (solid bar) for (a) Cr-fed rats $(n=8)$ and (b) controls $(n=9)$. The difference between Cr-fed rats and controls (c) is enlarged fivefold. The temporal resolution of the ADC determinations is $10 \mathrm{sec}$.

Details of the temporal evolution of diffusional changes are depicted in Figure 3. In particular, the $A D C_{A v}$ responses to transient ischemia may be characterized by three phases (I-III) and respective transition times $\left(\mathrm{t}_{1}, \mathrm{t}_{2}\right)$. Phase I lasts for about $1.5 \mathrm{~min}$ and accounts for about $20 \%$ of the total $A D C_{A v}$ reduction. It consists of an initial drop within the first 20-30 sec after occlusion and a somewhat slower diffusion change thereafter. The major part of the $A D C_{A V}$ reduction occurs during phase II and corresponds to about $60 \%$ of the total decrease. It may be described by an exponential decay during a period of approximately 2.5 min. The almost linear $A D C_{A v}$ decrease during phase III starts at 3.9 min after the onset of ischemia and contributes about $20 \%$ of the total change.

To quantify the dynamic diffusion changes, the $A D C_{A v}$ time courses were fitted by the superposition of a monoexponential and a linear decay representing phases II and III. The transition time $t_{1}$ between phases I and II is defined by the minimum difference between the model and the diffusi on data, whereas the transition time $t_{2}$ between phases II and III corresponds to the $5 \%$ level of the exponential decay. Pertinent values as well as decay time constants are summarized in Table 2. Changes between groups were not statisticall y significant. However, the transition from phase I to II as well as the time constant for the major $A D C_{A v}$ decay during acute ischemia were slightly prolonged in the Cr-fed group ( $23 \%$ increase of $\mathrm{t}_{1}, 10 \%$ increase of the decay time constant during phase II). This is al so shown in Fig. 3, which compares mean $A D C_{A v}$ time courses for $\mathrm{Cr}$-fed rats and controls.

During 60 min of reperfusion $A D C_{A v}$ values returned to preischemic levels in all cases. In fact, recovery was completed in shorter time periods as indicated by the $90 \%$ recovery time $t_{90}$ given in Table 2 . A Ithough the mean time courses of the $A D C_{A v}$ shown in Fig. 3 do not reveal major differences between $\mathrm{Cr}$-fed rats and controls, they suggest a slightly faster renormalization in the Cr-fed group during early reperfusion. This observation is supported by the $50 \%$ recovery time $t_{50}$ given in Table 2 . Again, differences between both groups were not statisti cally significant.

\section{DISCUSSION}

Although at the expense of high spatial resolution, the use of isotropically diffusion-weighted localized proton MRS of brain water yields quantitative determinations of the trace of the diffusion tensor at high temporal resolution and with excell ent signal to noise. In fact, the present data are characterized by a very low interindividual variation

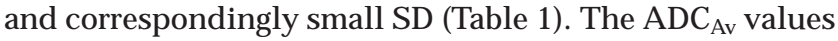
before ischemia and after reperfusion are in close agreement with results reported for cat and rat brain (9-11). Unfortunately, a more detailed comparison of absolute val ues as well as of changes during i schemi a with literature findings is hampered by differences in diffusion times,

Table 1

Mean Values of the Trace of $A D C_{A v}$ of Brain Water for Cr-Fed Rats and Controls*

\begin{tabular}{lcc}
\hline & $\begin{array}{c}\text { Control } \\
(n=9)\end{array}$ & $\begin{array}{c}\mathrm{Cr} \\
(n=8)\end{array}$ \\
\hline $\mathrm{ADC}_{\mathrm{Av}}\left(10^{-9} \mathrm{~m}^{2} \cdot \mathrm{sec}^{-1}\right)$ & & \\
$\quad$ Before ischemia & $0.78 \pm 0.03$ & $0.78 \pm 0.02$ \\
After $50-60$ min of reperfusion & $0.79 \pm 0.02$ & $0.79 \pm 0.03$ \\
$\Delta \mathrm{ADC}_{\mathrm{Av}}(\%)$ & & \\
After 12 min of ischemia & $-30.0 \pm 1.9$ & $-27.3 \pm 4.5$ \\
12 min after cardiac arrest & $-35.0 \pm 1.4$ & $-33.5 \pm 1.7$ \\
\hline
\end{tabular}

${ }^{*}$ Data given as means $\pm S D$. $A D C_{A v}$ values of individual animals represent time averages $(9 \mathrm{~min})$ obtained immediately before transient global ischemia and cardiac arrest, respectively. $\triangle A A_{A v}$ values denote the maximum $A D C_{A v}$ decrease after $12 \mathrm{~min}$ of transient ischemia or cardiac arrest. Differences between groups were not statistically significant (unpaired $t$ tests: Cr-fed rats vs. controls). 


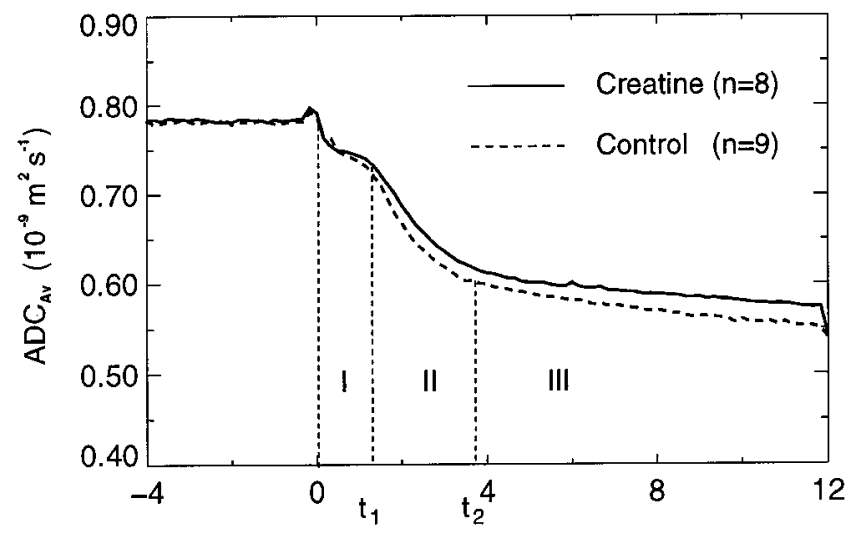

a time after occlusion ( $\mathrm{min})$

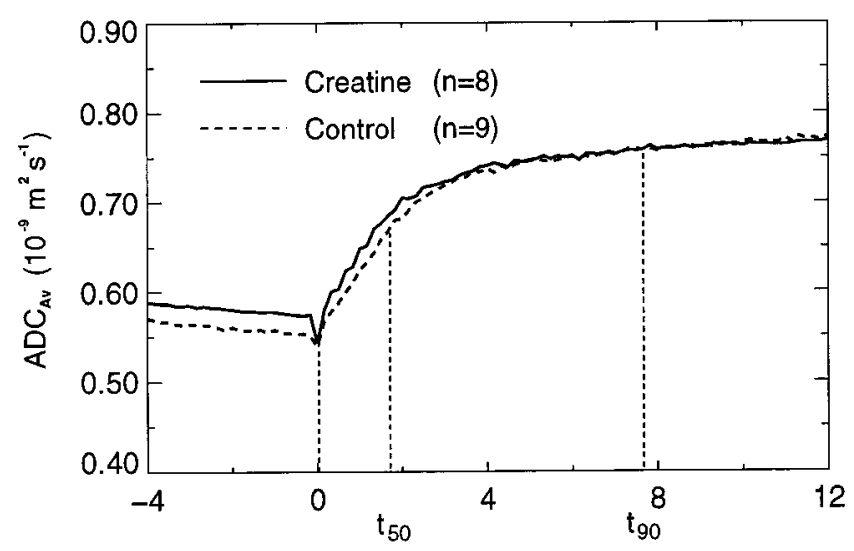

b

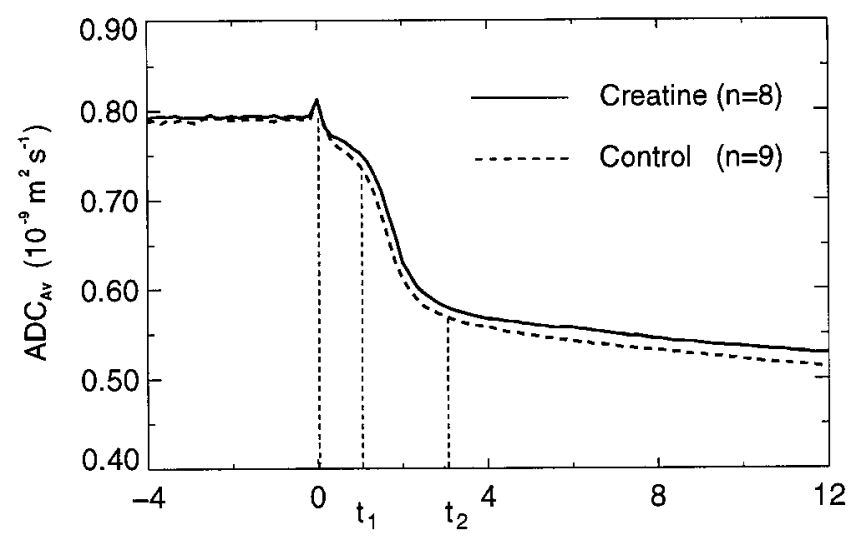

C

time post mortem (min)

FIG. 3. Expanded mean time courses of the trace of the brain water apparent diffusion coefficient tensor $\mathrm{ADC}_{\mathrm{Av}}$ in response to (a) transient global ischemia, (b) reperfusion, and (c) cardiac arrest for $\mathrm{Cr}$-fed rats (solid lines) and controls (dashed lines). The transition times $\left(t_{1}, t_{2}\right)$ and recovery times $\left(t_{50}, t_{90}\right)$ characterize the multiphasic diffusion changes during ischemia (and cardiac arrest) and reperfusion, respectively.

orientation of the diffusion gradients, brain regions, and species.

The temporal evolution of $A \mathrm{AC}_{\mathrm{Av}}$ changes in response to acute ischemia as well as after cardiac arrest exhibits a multiphasic behavior in agreement with previous observations by others ( $8-11)$. Thus, the early diffusion changes may be quantified in terms of transition times and decay time constants for a model comprising three phases of pathophysiologic events. Phase I is generally assumed to reflect the early breakdown of oxidative metabolism. For example, by use of biochemical methods it has been reported that both PCr and ATP pools are depleted after approximately $90 \mathrm{sec}$ of ischemia in adult rats (15). This timing is in excel lent agreement with the $t_{1}$ val ues obtained here (Table 2) for the transition of phase I to II. It further coincides with the timing reported for the increase of extracellular $\mathrm{K}^{+}$concentrations accompanying anoxic depolarization $(16,17)$. The subsequent strong $\mathrm{ADC}_{\mathrm{Av}}$ reduction during phase II $(\sim 2.5 \mathrm{~min})$ parallels the shift of extracellular to intracel lular water after the breakdown of energy supply and ion homeostasis. During phase III, i.e., $3.9 \mathrm{~min}$ after the onset of ischemia, the occurrence of a further but slower $A D C_{A v}$ decrease must be ascribed to brain cooling leading to a continuously decreasing tissue temperature (18).

During reperfusi on $\mathrm{ADC}_{\mathrm{Av}}$ val ues start to increase within a few seconds after the release of the balloon occluders. This observation is consistent with the immediate inflow of warm blood into the brain and a rapid recovery of the cerebral blood oxygenation. Both effects should account for a total $A D C_{A v}$ increase by approximately $40 \%$ of the $\triangle A D C_{A v}$ value. $A$ further increase should result from the normal isation of the $\mathrm{K}^{+}$concentration, which is completed within 4-5 min $(16,17)$. Typical recovery times are 1.5-1.8 $\min (50 \%)$ and approximately $8 \mathrm{~min}(90 \%)$, whereas full return to mean preischemic values requires 30-40 min (compare Fig. 2). Normalisation of the cerebral PCr/ATP ratio has been reported to take approxi mately $24 \mathrm{~min}$ after a 15-min period of transient gl obal ischemia (19).

Although diffusion changes after cardiac arrest follow the same pattern as during acute gl obal ischemia, they are slightly more pronounced ( $\triangle \mathrm{ADC}_{\mathrm{Av}}$ in Table 1$)$ and faster ( $\mathrm{t}_{1}$ and $t_{2}$ in Table 2). This may be explained by the fact that transient ischemia-in contrast to cardiac arrest-retains beneficial effects from residual collateral blood flow and a lower degree of brain cooling as a result of the isolating effect of the perfused skull.

Table 2

Transition Times $\left(t_{1}, t_{2}\right)$, Decay Time Constants (Phase II), and Recovery Times $\left(t_{50}, t_{90}\right)$ for Cr-Fed Rats and Controls*

\begin{tabular}{ccc}
\hline & $\begin{array}{c}\text { Control } \\
(n=9)\end{array}$ & $\begin{array}{c}\mathrm{Cr} \\
(n=8)\end{array}$ \\
\hline Ischemia & & \\
$t_{1}(I \rightarrow$ II) (min) & $1.3 \pm 0.4$ & $1.6 \pm 0.4$ \\
Time Constant (II) $\left(\mathrm{min}^{-1}\right)$ & $1.24 \pm 0.33$ & $1.36 \pm 0.31$ \\
$t_{2}(\mathrm{II} \rightarrow$ III) (min) & $3.9 \pm 0.8$ & $3.9 \pm 1.1$ \\
Reperfusion & $1.8 \pm 0.7$ & $1.5 \pm 0.5$ \\
$\quad t_{50}($ min) & $7.8 \pm 5.9$ & $8.6 \pm 3.5$ \\
$t_{90}($ min) & & \\
Cardiac arrest & $1.1 \pm 0.2$ & $1.2 \pm 0.1$ \\
$t_{1}(I \rightarrow$ II) (min) & $1.44 \pm 0.04$ & $1.45 \pm 0.02$ \\
Time constant (II) $\left(\right.$ min $\left.^{-1}\right)$ & $3.2 \pm 0.2$ & $3.3 \pm 0.1$ \\
$t_{2}(I I \rightarrow$ III) (min) &
\end{tabular}

*Values characterize the brain water $A D C_{A v}$ responses to transient global ischemia, reperfusion, and cardiac arrest. For a definition of phases and times, see Fig. 3. Differences between groups are not statistically significant (unpaired $t$ tests: Cr-fed rats vs. controls). 
In accordance with the aforementioned series of events, a putative neuroprotective role of $\mathrm{Cr}$ is mainly to be expected during the early phases of ischemia. Brain uptake of oral $\mathrm{Cr}$ is at least partially followed by phosphorylation and el evation of the cerebral PCr concentration, which may delay and/or attenuate ATP depletion during hypoxic/ischemic chal lenge (1-3,5,6). The use of high temporal resolution allowed us to identify mild trends for a prolonged phase I, a longer decay time constant during phase II (establishment of the cytotoxic edema), and a less severe total reduction of $\mathrm{ADC}_{\mathrm{Av}}$ in $\mathrm{Cr}$-fed rats versus controls. Although these findings suggest a slightly better performance of the brain energy status after oral administration of $\mathrm{Cr}$, larger beneficial effects are to be expected for less severe and/or focal hypoxic-ischemic challenges or in immature rats.

\section{REFERENCES}

1. Whittingham TS, Lipton P. Cerebral synaptic transmission during anoxia is protected by creatine. J Neurochem 1981;37:1618-1621.

2. Carter AJ, Müller RE, Pschorn U, Stransky W. Preincubation with creatine enhances levels of creatine phosphate and prevents anoxic damage in rat hippocampal slices. J Neurochem 1995;64:2691-2699.

3. Wilken B, Ramirez JM, Probst I, Richter DW, Hanefeld F. Creatine protects the central respiratory network of mammals under anoxic conditions. Pediatr Res 1998;43:8-14.

4. Michaelis T, Wick M, Fujimori H, Matsumura A, Frahm J. Proton MRS of oral creatine supplementation in rats. Cerebral metabol ite concentrations and ischemic challenge. NMR Biomed 1999, in press.

5. Matthews RT, Yang L, Jenkins BG, Ferrante RJ, Rosen BR, KaddurahDaouk R, Beal MF. Neuroprotective effects of creatine and cycl ocreatine in animal models of Huntington's disease. J Neurosci 1998;18:156-163.

6. Holtzman D, Togliatti A, Khait I, Jensen F. Creatine increases survival and suppresses seizures in the hypoxic immature rat. Pediatr Res 1998;44:410-414.

7. Dechent P, Pouwels PJW, Hanefeld F, Frahm J. Increase of total creatine in human brain after oral supplementation of creatine-monohydrate. Am J Physiol 1999, in press.

8. Davis D, Ulatowski J, Eleff S, Izuta M, Mori S, Shungu D, van Zijl PCM. Rapid monitoring of changes in water diffusion coefficients during reversible ischemia in cat and rat brain. Magn Reson Med 1994;31:454460.

9. Decanniere C, Eleff S, Davis D, van Zijl PCM. Correlation of rapid changes in the average water diffusion constant and the concentration of lactate and ATP breakdown products during global ischemia in cat brain. Magn Reson Med 1995;34:343-352.

10. Pierpaoli C, Alger JR, Righini A, Mattiello J, Dickerson R, Pres DD, Barnett A, Di Chiro G. High temporal resolution diffusion MRI of gl obal cerebral ischemia and reperfusion. J Cereb Blood Flow Metab 1996;16: 892-905.

11. Huang NC, Yongbi MN, Helpern JA. The influence of preischemic hyperglycemia on acute changes in the apparent diffusion coefficient of brain water gl obal ischemia in rats. Brain Res 1997;757:139-145.

12. Hossmann KA, Hoehn-Berlage M. Diffusion and perfusion MR imaging of cerebral ischemia. Cerebrovasc Brain Metab Rev 1995;7:187-217.

13. Wick M, Fujimori $\mathrm{H}$, Michaelis T, Frahm J. Oral creatine and brain water ADC during transient gl obal ischemia in rat brain. In: Proceedings of the 6th Annual Meeting of the ISMRM, Sydney, 1998. p 1766.

14. Mori S, van Zijl PCM. Diffusion weighting by the trace of the diffusion tensor within a single scan. Magn Reson Med 1995;33:41-52.

15. Katsura K, Rodriguez de Turco EB, Folbergrová J, Bazan NG, Siesjö BK. Coupling among energy failure, loss of ion homeostasis, and phospholipase $A_{2}$ and C activation during ischemia. J Neurochem 1993;61:16771684.

16. Hansen AJ, Gjedde A, Siemkowicz E. Extracellular potassium and blood flow in the post-ischemic rat brain. Pflugers A rch 1980;389:1-7.

17. Ekholm A, Katsura K, Kristián T, Liu M, Folbergrová J, Siesjö BK. Coupling of cellular energy state and ion homeostasis during recovery following brain ischemia. Brain Res 1993;604:185-191.

18. Hasegawa Y, Latour LL, Sotak CH, Dardzinski BJ, Fisher M. Temperature dependent changes of the apparent diffusion coefficient of water in normal and ischemic brain of rats. J Cereb Blood Flow Metab 1994;14: 383-390.

19. de la Torre JC, Saunders J, Fortin T, Butler K, Richard M. Return of ATP/PCr and EEG after $75 \mathrm{~min}$ of global brain ischemia. Brain Res 1991;542:71-76. 